\title{
Genetic Algorithms in Model Structure Identification for Fuel Cell Polarization Curve
}

\author{
Markku Ohenoja, Aki Sorsa, and Kauko Leiviskä
}

\begin{abstract}
Evolutionary optimizers, such as genetic algorithms, have earlier been successfully applied to find the parameter values for the fuel cell polarization curve models. The structure of these, typically semi-empirical, models have evolved during the decades. In this study, the model structures were reviewed and a new model structure was generated. Genetic algorithms were used to determine the optimized model structure with linear model parameters. Four different fuel cells, one with varying operating conditions, were studied. The results show that the model can outperform the semiempirical model utilized in number of studies without increasing the model complexity.
\end{abstract}

\section{INTRODUCTION}

Numerical modeling of polymer electrolyte membrane fuel cell (PEM-FC) stacks allows investigation of the effect of system parameters, such as different configurations and operating conditions [1]. FC models often have modular structure with separate modules for electrochemical phenomena, energy balance, and mass balances. The Membrane-Electrode Assembly (MEA) and therefore FC's electrochemical behavior (in the steady-state) can efficiently be described with an equivalent-circuit model determining the polarization curve of the FC. Typically, these models have also mechanistic background, and can therefore be classified as semi-empirical models. This kind of simplified approach enables to utilize the model in a complete fuel cell power system simulation, where utilization of rigorous first principle (mechanistic) models may often be too difficult [1]. The semi-empirical model structures proposed for the FC polarization curve are reviewed in Section II. Other approaches to FC modeling involve rigorous mechanistic models [2] and purely empirical (data-driven) models [3].

The power of different evolutionary optimizers in the parameter estimation problem for the semi-empirical FC models has already been shown, see e.g. $[4,5,6,7,8]$. In this study, a novel approach to apply the evolutionary optimization tools in model structure identification of the FC polarization curve model is taken. The aim is to derive a FC polarization curve model with linear coefficients with respect to its parameters. The proposed model structure is validated for different fuel cells and operating conditions and the applicability of the model is discussed.

\section{REVIEW OF POLARIZATION CURVE MODEL STRUCTURES}

The steady-state electrochemical model of a PEM-FC aims to describe the thermodynamic potential (or the open-

All authors are with the Control Engineering Research Group, University of Oulu, P.O.Box 4300, 90014 Oulu, Finland (corresponding author: Markku Ohenoja, phone: +358-29-448-2473; e-mail: markku.ohenoja@oulu.fi). circuit voltage) and the different losses (or overvoltage) in the performance. The basis for the semi-empirical model approach is the model presented in [9]. In this model, two loss terms are accounted for: 1$)$ the Tafel slope $\left(a_{1}\right)$ describing the activation overvoltage governing in low currents, and 2) the Ohmic overvoltage being a linear function of the current:

$$
V=E_{0}-a_{1} \log (i)-a_{2} i
$$

where $V$ is the $\mathrm{FC}$ voltage, $E_{0}$ is the internal (or reversible, thermodynamic) potential of the $\mathrm{FC}, i$ is the FC current, and $a_{1}, a_{2}$ are the model parameters. In [10], the same authors added a third loss term describing the mass transport limitations in high currents. This concentration overvoltage term was given as an exponential function with respect to FC current:

$$
V=E_{0}-a_{1} \log (i)-a_{2} i-a_{3} \exp \left(a_{4} i\right)
$$

where $a_{3}$ and $a_{4}$ are the model parameters for the exponential term. However, this addition has no physicochemical background and can therefore be considered as an attempt to fit the experimental data [11]. In [1], the model and its curve fitting capabilities were further developed by adding a logarithmic pressure ratio term:

$$
V=E_{0}-a_{1} \log (i)-a_{2} i-a_{3} \exp \left(a_{4} i\right)-a_{5} \log \left(\frac{p}{p_{O 2}}\right)
$$

where $a_{5}$ is the additional model parameter, $p$ is the FC total pressure, and $p_{\mathrm{O} 2}$ is the partial pressure of oxygen in the cathode side. Squadrito et al. [12] have modified the concentration overvoltage term and introduced it as a logarithmic function of the ratio between the current and the limiting current:

$$
V=E_{0}-a_{1} \log (i)-a_{2} i+a_{3} i^{a_{4}} \log \left(1-\frac{i}{i_{\mathrm{lim}}}\right)
$$

where $a_{1}, a_{2}, a_{3}$ and $a_{4}$ are the model parameters and $i_{\text {lim }}$ is the limiting current where fuel is supplied at its maximum rate. This loss term is also present in many later suggested model structures. For example, in [13] an effort to explain all the model parameters with physical origin and meaning was made. Kulikovsky et al. [14] developed an analytical model for the cathode side losses, having the limiting current expressed in a logarithmic term, as well.

Simultaneously to studies mentioned above, a steadystate electrochemical model [15] and a generalized steadystate electrochemical model (GSSEM) [11] for a PEM-FC have been developed. In these studies, the loss terms of the polarization curve model were decomposed to have physical 
meaning (and therefore neglect exponential and pressure ratio terms). The model consists of basic expression for a single cell:

$$
V=E_{0}-\eta_{a c t, a}-\eta_{a c t, c}-\eta_{o h m}
$$

where the terms describe the internal potential, the anode overvoltage $\left(\eta_{\text {act, }, a}\right)$, the cathode overvoltage $\left(\eta_{\text {act }, c}\right)$, and Ohmic overvoltage $\left(\eta_{\text {ohm }}\right)$, respectively. The internal potential is described with the Nernst equation:

$$
\begin{aligned}
E_{0}= & 1.229-0.85 \cdot 10^{-3}(T-298.15) \\
& +4.3085 \cdot 10^{-5} T \ln \left(p_{H_{2}} p_{O 2}^{0.5}\right)
\end{aligned}
$$

where $T$ is the operation temperature, and $p_{H 2}$ is the hydrogen partial pressure in anode side. The activation overvoltage can be lumped to a single expression:

$$
\eta_{a c t}=a_{1}+a_{2} T+a_{3} T \ln \left(C_{O 2}\right)+a_{4} T \ln (i)
$$

where $a_{1}, a_{2}, a_{3}$ and $a_{4}$ are the model parameters, and $C_{O 2}$ is the oxygen concentration in the cathode side. Mann et al. [11] shows how the model parameters can be determined from theoretical equations with kinetic, thermodynamic and electrochemical foundations. In (6) and (7), the effect of the operating conditions is taken into account. The partial pressures and the oxygen concentration can be calculated as a function of the anode and cathode inlet pressures $\left(p_{a}, p_{c}\right)$, temperature, relative humidity of vapor in the anode and cathode $\left(R H_{a}, R H_{c}\right)$, saturation pressure of the water vapor $\left(p^{\text {sat }}{ }^{2 O}\right)$, effective electrode area $(A)$, and cell current. The equations are as follows [4]:

$$
\begin{aligned}
& \log _{10}\left(p_{H 2 O}^{\text {sat }}\right)=-2.18+2.95 \cdot 10^{-2}(T-273.15) \\
& -9.18 \cdot 10^{-5}(T-273.15)^{2}+1.44 \cdot 10^{-7}(T-273.15)^{3}
\end{aligned}
$$

$$
\begin{gathered}
p_{O 2}=\left(R H_{c} p_{H 2 O}^{\text {sat }}\right)\left\{\left[\exp \left(\frac{4.192\left(\frac{i}{A}\right)}{T^{1.334}}\right)\left(\frac{R H_{c} p_{H 2 O}^{s a t}}{P_{c}}\right)\right]^{-1}-1\right\}_{(9)} \\
p_{H 2}=\frac{R H_{a} p_{H 2 O}^{s a t}}{2}\left\{\left[\exp \left(\frac{1.635\left(\frac{i}{A}\right)}{T^{1.334}}\right)\left(\frac{R H_{a} p_{H 2 O}^{s a t}}{P_{a}}\right)\right]^{-1}-1\right\}_{(10)} \\
C_{O 2}=\frac{p_{O 2}}{5.08 \cdot 10^{6} \exp (-498 / T)}
\end{gathered}
$$

The Ohmic overvoltage is described with Ohm's law equations. In [15], the Ohmic losses were described as a function of $T$ and $i$ with an empirical model with quadratic effects:

$$
\eta_{o h m}=a_{5}+a_{6} T+a_{7} i+a_{8} T i+a_{9} T^{2}+a_{10} i^{2}
$$

However, they also mentioned that logarithmic, exponential or other kind of relationships might be necessary. In [11], constant, but unknown resistances to electron flow were assumed and the empirical correlation of resistances to proton flow was given as:

$$
\eta_{\text {ohm }}=i \cdot\left(a_{5}+\frac{r_{m} l}{A}\right)
$$

where $a_{5}$ is the model constant for the electronic resistances (usually indicated as $R_{e}$ or $R_{c}$ ) and fractional term describes the proton resistances $\left(R_{p}\right)$ as function of the membrane specific resistivity $r_{m}$, membrane thickness $l$ and cell active area $A$. For the Nafion membranes, the $r_{m}$ can be expressed as a function of $T$ and $i$ as follows:

$$
r_{m}=\frac{181.6 \cdot\left[1+0.03(i / A)+0.062(T / 303)(i / A)^{2.5}\right]}{\left[a_{6}-0.634-3(i / A)\right] \cdot \exp \left[4.18\left(T^{T-303 / T}\right)\right]}
$$

where $a_{6}$ is the adjustable fitting parameter (typically indicated as $\lambda$ or $\beta$ ). Hence, there are six model parameters in the GSSEM by Mann et al. The studies focusing on simulations of FC systems and experimental parameter fitting of polarization curve models typically involve the GSSEM and the concentration overvoltage term $\left(\eta_{c o n}\right)$, as well:

$$
V=E_{0}-\eta_{a c t, a}-\eta_{a c t, c}-\eta_{o h m}-\eta_{c o n}
$$

For example, in [4,16], $\eta_{c o n}$ is given as in (4), but with one lumped parameter:

$$
\eta_{c o n}=a_{7} \ln \left(1-\frac{i}{i_{\mathrm{lim}}}\right)
$$

where $a_{7}$ is the model parameter (typically indicated with $B)$. Hence, this modified model consists of seven unknown parameters. There are also further modifications to the concentration overvoltage term, but those are not described here. In [17], ageing terms were introduced for the GSSEM. The FC performance degradation rate was expressed in conjunction with the equation for $a_{2}$ in (7).

As should be obvious by now, the FC electrochemical models have physical background, but the problem can be made easier with parameterized models, which can be tuned with the measurable variables from the operating fuel cells. With the aid of experimental data, the models can be altered to give better prediction abilities for the inspected FC system (curve fitting properties), but with the expense of losing the generalization abilities for the unseen FC systems.

\section{ALGORITHM FOR MODEL STRUCTURE IDENTIFICATION}

The model structure identification is a difficult task including the selection of model variables and mathematical operations. Intelligent methods, such as genetic algorithms [18], can be applied to find an optimized nonlinear model structure, whenever reasonable amount of experimental data is available. An example is given in [19], where feature selection, mathematical operators for the selected features, and the arithmetic operations between the generated terms were encoded to chromosomes in order to facilitate model structure optimization with genetic algorithms. Here, the same approach is used for FC polarization curve optimization. 


\section{A. Inspected data}

The polarization curve data is originated from $[4,20]$ comprising four different fuel cells. The numerical data is extracted from the plotted polarization curves by careful visual inspection. For the three data sets presented in [20], ten current-voltage pairs are available. The data sets presented in [4] consists of four polarization curves for one FC observed in different operating conditions. Each data set has 15 current-voltage pairs. In addition to current-voltage values, nominal operating conditions (temperature, partial pressures of oxygen and hydrogen) and cell properties (cell active area, membrane thickness, limiting current) are given and used as an input data for the model identification. Equations (8-11) can be used to calculate the partial pressures of oxygen and hydrogen, and anode oxygen concentration, respectively. Hence, the total number of current-voltage pairs, $k$, is 90 .

\section{B. Model structure encoding to genetic algorithms}

Fig. 1 depicts how the variable selection and operators are encoded. First, the number of possible model variables $(j$, according to the data set $\left.y(k), x_{j}(k)\right)$ are defined and corresponding number of bits are reserved in order to be able to describe the model variables in binary format. Then, the allowable maximum number of terms to be used in the model structure $(n)$ is defined. This increases the chromosome size by propagating the number of reserved bits to account for the allowable number of variables. Next, the number of mathematical transformation operators to be tested is defined and corresponding number of bits is again reserved to describe operator for each selected variable. Similarly, the available uniting operators need to be described in binary format and correct number of bits need to be reserved in order to describe the mathematical operator between the selected variables. An example of the resulted chromosome is presented in Fig 2.

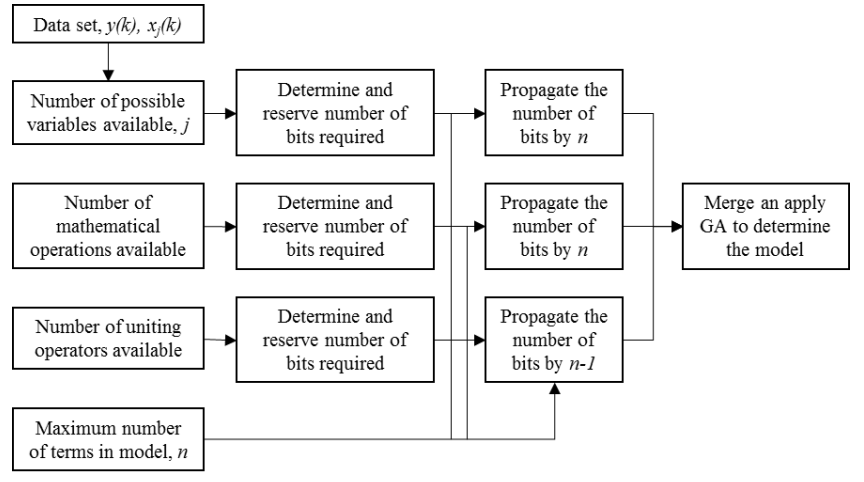

Figure 1. Encoding procedure.

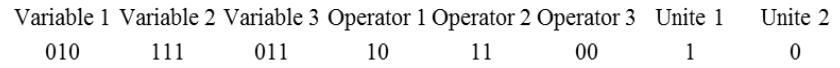

Figure 2. Example of binary coded chromosome for three term model structure $(n=3)$ with up to eight possible variables $\left(2^{3}\right)$, four possible transformation operators $\left(2^{2}\right)$, and two possible uniting operators $\left(2^{1}\right)$.

The genetic algorithm uses a population of randomly generated chromosomes and performs crossover and mutation operations until the convergence criterion is met or the selected number of generations is performed. The resulting best individual contains a vector of bits, which can be interpreted into a model with the following general structure:

$$
\hat{y}=a_{0}+a_{1} f_{1}(+/ \times / \div / \wedge) a_{2} f_{2}(+/ \times / \div / \wedge) . . a_{n} f_{n}
$$

where, $\dot{y}$ is the predicted FC voltage, $f_{1} \ldots f_{\mathrm{n}}$ are the selected variables among $x_{j}$ with their mathematical transformations and $a_{0} \ldots a_{\mathrm{n}}$ are the regression coefficients. The model may be nonlinear due to the mathematical operators applied to the model variables. However, the model is always linear in respect to the regression coefficients and thus it can be solved as an ordinary regression equation. Depending on the uniting mathematical operators, the model may have a smaller amount of regression coefficients than the selected variables as the terms are fused together.

From the inspected data sets, $i, T, p_{\mathrm{O} 2}, p_{\mathrm{H} 2}, \mathrm{C}_{\mathrm{O} 2}$, $i_{\text {lim }}$, are selected as available model variables. Hence, three bits are needed to represent all the candidates. With three bits, eight candidates could be used $(j=8)$. Therefore, $i / i_{\text {lim }}$ is used as an additional model variable candidate. The eighth variable is the regression constant. The model structure inspected in numerous studies (e.g. [4,5,6]) consist of seven unknown parameters. Hence, without increasing the model complexity, seven terms are allowed for the optimized model structure, as well. The available functional transformations (mathematical operations) applied to each variable are $(1, \wedge 2, \wedge 3, \operatorname{sqrt}, \exp (-$ ), 1/, exp, and ln). Again, three bits are required to describe these eight options. The uniting mathematical operations $\left(+,{ }^{*}, / \wedge\right)$ can be described with two bits. With seven terms, only six convolutions are needed. In total, $7 *(3+3)+6 * 2=$ 52 bits are needed to describe one candidate model structure within the GA.

In the GA, uniform crossover and mutation operations are applied. Tournament selection is used with the number of candidates set to 5 . In order to avoid the very best solution disappearing from the population, elitism is applied by replacing the worst candidate from the new generation with the best candidate from the previous generation. The algorithm used a population size of 500, number of generations were 100 , crossing probability was 0.9 , and the mutation probability was 0.05 . The optimization loop involved 100 repetitions. The coding and optimization was performed with Matlab® software. The random number generator was initialized prior to the loop in order to be able to repeat the results.

\section{Parameter estimation and objective function}

The candidates provided by the GA are evaluated after the parameter estimation of the regression coefficients. Since the parameter estimation problem is linear, the regression coefficients are the least squares solution of (17):

$$
\left[\begin{array}{c}
a_{1} \\
\vdots \\
a_{n}
\end{array}\right]=\left[\begin{array}{ccc}
f_{1}(1) & \ldots & f_{n}(1) \\
\vdots & \ddots & \vdots \\
f_{1}(k) & \ldots & f_{n}(k)
\end{array}\right]^{-1}\left[\begin{array}{c}
y(1) \\
\vdots \\
y(k)
\end{array}\right]
$$

The regression coefficients are calculated independently for different fuel cells. The objective function is the sum of squared errors (SSE) between the experimental data and the predicted data from the candidate model comprising all the available FC data sets: 


$$
S S E=\sum_{F C} \sum_{k}(y-\hat{y})^{2}
$$

\section{RESULtS}

Model structure identification is based on the data from three fuel cells (Ballard, BCS, SR-12) with 10 samples, and four data sets from the fourth fuel cell $(250 \mathrm{~W})$ with total of 60 samples. Linear regression parameters are calculated for each cell. For the fourth cell, only data sets 1 and 2 are utilized in parameter estimation. Data sets 3 and 4 are left for model validation. Their predicted values are based on the regression parameters estimated with data sets 1 and 2, and incorporated in the objective function calculation. This division allows the model have some generalization ability for different fuel cells and for different operation conditions in one cell.

The optimization results are presented in Fig. 3, where the seven predicted polarization curves with experimental data are shown. Clearly, the model can follow the experimental data very accurately. In Table 1, the SSE values for each case is given. In comparison, the SSE values from our earlier studies [21,5] are given. It should be noted, that all these use exactly the same data sets and comparison of SSE values is straightforward. The model structure used in $[5,21]$ is the one presented in $[4,16]$.

The results in Table 1 show that for the SR-12 and Ballard fuel cell polarization curves, the optimized model structure produces better results than the model structure in [21] in terms of SSE. For the BCS polarization curve, a better fit was found in [21]. Based on the very low SSE values, acceptable result is achieved in both studies for these three fuel cells. For the $250 \mathrm{~W}$ fuel cell comprising data in varying operating conditions, the optimized model structure can fit the data with a significant lower SSE than in [5]. In [5], the best fit showed a SSE value of 8.4854 , whereas this model achieves a SSE of 1.6154.

TABLE I. MODEL PERFORMANCE FOR THE STUDIED FUEL CELLS

\begin{tabular}{|l|c|c|c|}
\hline \multirow{2}{*}{ Fuel cell } & \multicolumn{3}{|c|}{ SSE } \\
\cline { 2 - 4 } & Optimized model structure & {$[21]$} & [5] \\
\hline $250 \mathrm{~W} / 1$ & 0.2384 & & \\
\hline $250 \mathrm{~W} / 1$ & 0.2782 & & \\
\hline $250 \mathrm{~W} / 1$ & 0.2059 & & \\
\hline $250 \mathrm{~W} / 1$ & 0.8929 & & \multirow{2}{*}{} \\
\hline $250 \mathrm{~W} / \mathrm{all}$ & 1.6154 & 0.4475 & \\
\hline SR-12 & 0.0615 & 0.1040 & \\
\hline BCS & 0.2148 & 0.0918 & \\
\hline Ballard & 0.0640 & & \\
\hline
\end{tabular}

In order to complete the comparison, several authors have used the same semi-empirical model structure and have been able to find better SSE values than reported in [5]. For example, Sun et al. [6] managed to reach SSE value of 7.99 for the $250 \mathrm{~W}$ fuel cell. However, the data sets are not exactly the same, although interpreted from the same original polarization curves. Therefore, direct comparison of SSEs between the studies cannot be made. Further, Yang et al. [22] reached a low SSE value of 1.1746, but the model structure was altered by adding three more free parameters to the modeling and parameter fitting. The model structure found in this study provides a very low SSE values with the same number of free parameters, and therefore with comparable model complexity than the semi-empirical model structure used in $[4,5,6,16,21]$. Naturally, part of the difference in the observed SSE values can be explained with the constrained parameter values of semi-empirical model. It was shown in [5], that the expanded search range leads to more accurate results. With the approach taken here, the optimization problem is unconstrained.

The optimized model structure found for the FC polarization curve can be written as:

$$
\hat{y}=a_{0}+a_{1,2} f_{1}^{\wedge} f_{2}+a_{3} f_{3}+a_{4} f_{4}+a_{5,6} f_{5} \div f_{6}+a_{7} f_{7}
$$

where

$$
\begin{aligned}
& f_{1}=\frac{1}{C_{02}}, f_{2}=\frac{i}{i \lim }, f_{3}=10^{i / i \lim }, f_{4}=p_{H 2}, \\
& f_{5}=\sqrt{\frac{i}{i \lim }}, f_{6}=T, f_{7}=10^{-i / i \lim }
\end{aligned}
$$

From (21), it can be seen that the term $i / i_{\text {lim }}$ is repeated several times in the model. Indeed, $i_{\text {lim }}$ has a strong effect on the polarization curve as it determines the end point for the curve. In this exercise, it was assumed that the value for the $i_{\text {lim }}$ is known and fixed throughout the operation conditions. Naturally, unknown $i_{\text {lim }}$ would require new model structure identification. In addition, the membrane properties (at least the membrane thickness) could be added to the available variable list prior to model structure optimization.

The approach taken in this study is fundamentally datadriven. However, typically the data-driven methods use rather vast amount of (measured) data and data preprocessing. In this exercise, the data were very limited with only $V, p_{\mathrm{O} 2}, p_{\mathrm{H} 2}, \mathrm{C}_{\mathrm{O} 2}$ and $i / i_{\text {lim }}$ changing as a function if $i$ and the operating conditions $\left(T, P_{a}, P_{c}\right)$. The values for $p_{O 2}, p_{H 2}$, $\mathrm{C}_{\mathrm{O} 2}$ were calculated from the semi-empirical equations (811). Despite the limited amount of data, the model structure established was found to have (limited) generalization ability. Although the model was efficiently fitted to four different fuel cell polarization curves, a more rigorous benchmark test with rich data set are needed. Data originated from $[4,20]$ are only one example with the limited amount of data points. Benchmark data could be, for example, simulated via rigorous model in different operating condition, and adding noise and drift elements. Only with such data can the model performance be efficiently tested. Although the application of data-driven models is restricted and best suited to fuel cell studies with existing equipment, data-driven models can be feasible in diagnostics purposes, control designs and large-scale simulations. More rigorous mechanistic models should be preferred when the focus is on new designs. 

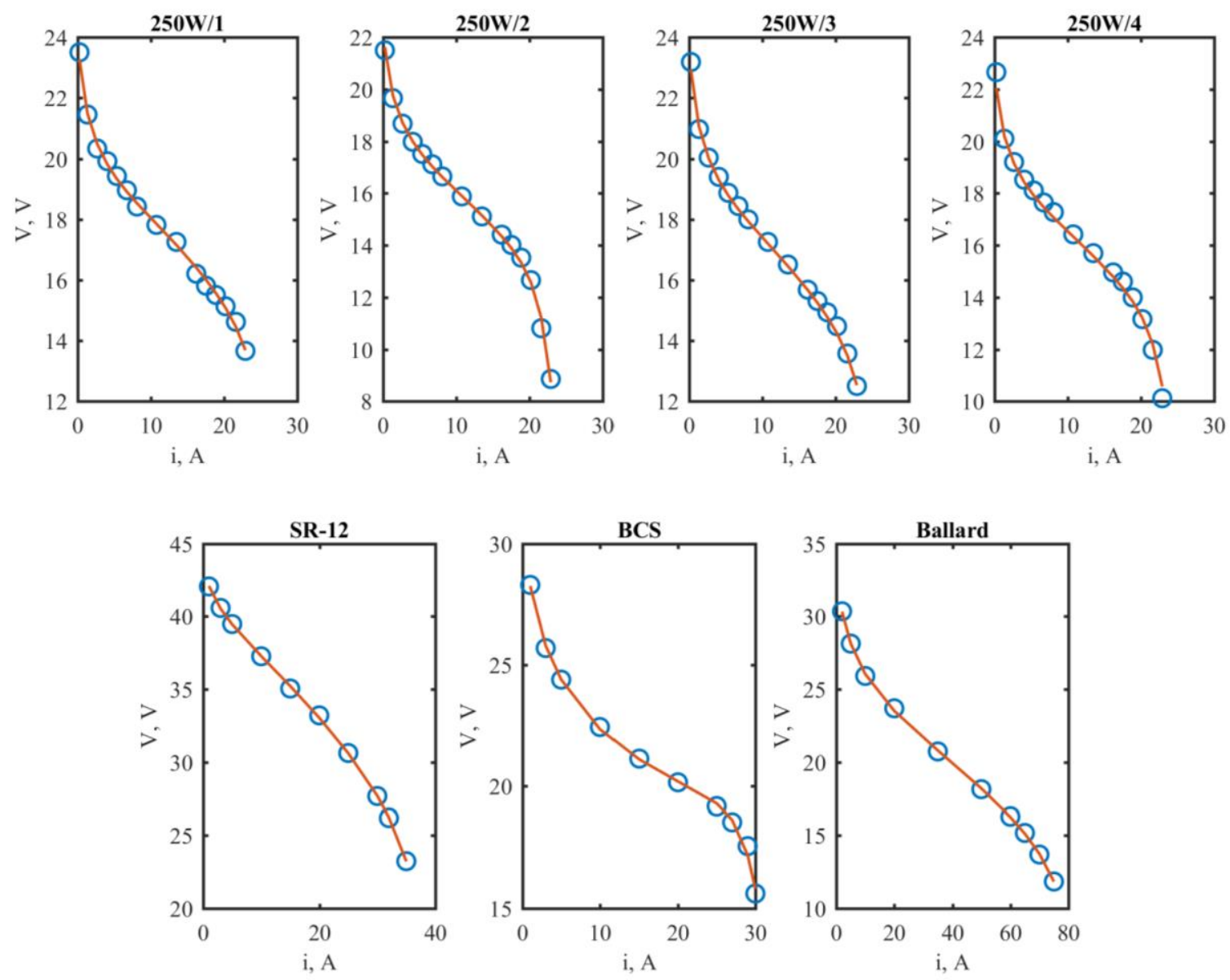

Figure 3. Polarization curves with the optimized model structure (solid line) and the experimental data (circles) interpreted from [4,20].

\section{CONCLUSION}

An application of utilizing Genetic Algorithms in model structure identification was presented. The procedure was successfully applied to determine an optimized model structure for PEM-FC polarization curve with relative low model complexity. In terms of fit to the experimental data, the model outperformed the semi-empirical model structure utilized in number of studies. The results also indicated generalization ability with respect to different fuel cells and operating conditions, but proper benchmark data are required for more comprehensive tests and to draw solid conclusions.

\section{REFERENCES}

[1] J. H. Lee, T. R. Lalk, and A. J. Appleby, "Modeling electrochemical performance in large scale proton exchange membrane fuel cell stacks," Journal of Power Sources, vol. 70, pp. 258-268, 1998.

[2] A.A. Shah, K.H. Luo, T.R. Ralph, F.C. Walsh, "Recent trends and developments in polymer electrolyte membrane fuel cell modelling", Electrochimica Acta, vol. 56, pp. 3731-3757, 2011.

[3] I.-S. Han, C.-B. Chung, "Performance prediction and analysis of a PEM fuel cell operating on pure oxygen using data-driven models: A comparison of artificial neural network and support vector machine" International Journal of Hydrogen Energy, vol. 41, pp. 10202-10211, 2016.
[4] Z.-J. Mo, X.-J. Zhu, L.-Y. Wei, and G.-Y. Cao, "Parameter optimization for a PEMFC model with a hybrid genetic algorithm," International Journal of Energy Research, vol. 30, pp. 585-597, 2006.

[5] M. Ohenoja and K. Leiviskä, "Validation of genetic algorithm results in a fuel cell model," International Journal of Hydrogen Energy, vol. 35, no. 22, pp. 12618-12625, 2010.

[6] Z. Sun, N. Wang, Y. Bi, and D. Srinivasan, "Parameter identification of PEMFC model based on hybrid adaptive differential evolution algorithm," Energy, vol. 90, pp. 1334-1341, 2015.

[7] O. E. Turgut and M. T. Coban, "Optimal proton exchange membrane fuel cell modelling based on hybrid Teaching Learning Based Optimization - Differential Evolution algorithm," Ain Shams Engineering Journal, vol. 7, no. 1, pp. 347-360, 2016.

[8] A. A. El-Fergany, "Extracting optimal parameters of PEM fuel cells using Salp Swarm Optimizer," Renewable Energy, vol. 119, pp. 641648, Apr. 2018.

[9] S. Srinivasan, E.A. Ticianelli, C.R. Derouin, and A. Redondo, "Advances in solid polymer electrolyte fuel cell technology with low platinum loading electrodes", Journal of Power Sources, vol. 22, pp. 359-375, 1988

[10] J. Kim, S.-M. Lee, S. Srinivasan, and C.E. Chamberlin, "Modeling of Proton Exchange Membrane Fuel Cell Performance with an Empirical Equation," Journal of The Electrochemical Society, vol. 142, pp. 2670, 1995.

[11] R. F. Mann, J. C. Amphlett, M. A. I. Hooper, H. M. Jensen, B. A Peppley, and P. R. Roberge, "Development and application of a generalised steady-state electrochemical model for a PEM fuel cell," Journal of Power Sources, vol. 86, pp. 173-180, 2000.

[12] G. Squadrito, G. Maggio, E. Passalacqua, F. Lufrano, and A. Patti, "An empirical equation for polymer electrolyte fuel cell (PEFC) behaviour," Journal of Applied Electrochemistry, vol. 29, pp. 14491455,1999 
[13] L. Pisani, G. Murgia, M. Valentini, and B. D'Aguanno, "A new semiempirical approach to performance curves of polymer electrolyte fuel cells," Journal of Power Sources, vol. 108, pp. 192-203, 2002.

[14] A. A. Kulikovsky, T. Wüster, A. Egmen, and D. Stolten, "Analytical and Numerical Analysis of PEM Fuel Cell Performance Curves," Journal of The Electrochemical Society, vol. 152, pp. A1290, 2005.

[15] J. C. Amphlett, R.M. Baumert, R.F. Mann, B.A. Peppley, P.R. Roberge, and T.J. Harris, "Performance Modeling of the Ballard Mark IV Solid Polymer Electrolyte Fuel Cell," Journal of The Electrochemical Society, vol. 142, pp. 1, 1995.

[16] J. M. Correa, F. A. Farret, L. N. Canha, and M. G. Simoes, "An electrochemical-based fuel-cell model suitable for electrical engineering automation approach," IEEE Transactions on Industrial Electronics, vol. 51, pp. 1103-1112, 2004.

[17] M. W. Fowler, R. F. Mann, J. C. Amphlett, B. A. Peppley, and P. R. Roberge, "Incorporation of voltage degradation into a generalised steady state electrochemical model for a PEM fuel cell," Journal of Power Sources, vol. 106, pp. 274-283, 2002.

[18] Z. Michalewicz, Genetic Algorithms + Data Structures = Evolution Programs. Berlin, Heidelberg: Springer Berlin Heidelberg, 1996.

[19] A. Sorsa, M. Ruusunen, K. Leiviskä, S. Santa-Aho, M. Vippola, and T. Lepistö, "An Attempt to Find an Empirical Model between Barkhausen Noise and Stress," Materials Science Forum, vol. 768 769, pp. 209-216, 2013.

[20] X. D. Xue, K. W. E. Cheng, and D. Sutanto, "Unified mathematical modelling of steady-state and dynamic voltage-current characteristics for PEM fuel cells," Electrochimica Acta, vol. 52, pp. 1135-1144, 2006.

[21] M. Ohenoja and K. Leiviska, "Identification of electrochemical model parameters in PEM fuel cells," in 2009 International Conference on Power Engineering, Energy and Electrical Drives, pp. 363-368, 2009.

[22] S. Yang, R. Chellali, X. Lu, L. Li, and C. Bo, "Modeling and optimization for proton exchange membrane fuel cell stack using aging and challenging P systems based optimization algorithm," Energy, vol. 109, pp. 569-577, 2016. 